\title{
Publisher Correction: High-speed, cortex-wide volumetric recording of neuroactivity at cellular resolution using light beads microscopy
}

Jeffrey Demas, Jason Manley, Frank Tejera, Kevin Barber, Hyewon Kim, Francisca Martínez Traub, Brandon Chen (D) and Alipasha Vaziri (i)

Correction to: Nature Methods https://doi.org/10.1038/s41592-021-01239-8, published online 30 August 2021.

In the version of this Article originally published, Supplementary Videos 4-12 appeared out of order. The current Supplementary Video 4 initially appeared as Supplementary Video 12, while the current Supplementary Videos 5-12 initially appeared as Supplementary Videos 4-11. Further, a reader identified that a light beam was missing in the Extended Data Fig. $1 \mathrm{~b}$ diagram, now appearing horizontally between mirrors 12 and 9.

The changes have been made to the online version of the article.

Published online: 8 November 2021

https://doi.org/10.1038/s41592-021-01337-7

(c) The Author(s), under exclusive licence to Springer Nature America, Inc. 2021 